\title{
Oxidation of metal thin films by atomic oxygen: a Low Energy Ion Scattering study
}

\author{
C. R. Stilhano Vilas Boas ${ }^{\mathrm{a}, *}$, J. M. Sturm ${ }^{\mathrm{a}}$, F. Bijkerk ${ }^{\mathrm{a}}$ \\ ${ }^{a}$ Industrial Focus Group XUV Optics, MESA+ Institute for Nanotechnology, University of \\ Twente, Enschede, The Netherlands
}

\begin{abstract}
In this study, we combine Low Energy Ion Scattering (LEIS) static and sputter depth profiles for characterization of the oxidation kinetics on $\mathrm{Zr}$, Mo, $\mathrm{Ru}$ and Ta films of various thicknesses, following exposure to atomic oxygen at room temperature $\left(\sim 20^{\circ} \mathrm{C}\right)$. A method for non-destructive determination of the oxide growth rate via LEIS static depth profiling (static DP) is presented in detail. This method shows high sensitivity to the oxide thickness formed, and the results are in agreement with those obtained by X-ray reflectometry (XRR) and sputter depth profiling (sputter DP). Sequential exposures of oxygen isotopes in combination with LEIS sputter DP are applied to elucidate the growth mechanism of the oxide films. The results indicate that the oxidation kinetics at the applied experimental conditions is directly influenced by the metal work function, characterizing a Cabrera-Mott growth type. The maximum thickness of the formed oxide and oxide growth rate are in the order: $\mathrm{Zr} \approx \mathrm{Ta}>\mathrm{Mo}>\mathrm{Ru}$. The combining of analysis by LEIS static DP and isotope tracing sputter DP is decisive in the characterization of oxidation kinetics in the room temperature regime.

Keywords: Low Energy Ion Scattering, Room temperature oxidation, Atomic oxygen, Oxidation kinetics

*Corresponding author

Email address: c.r.stilhanovilasboas@utwente.nl (C. R. Stilhano Vilas Boas),
\end{abstract}

\section{Introduction}

The oxidation of surfaces represents a complex reaction scenario in the field of thin films. Regarding transition metals and their oxides, the 
understanding of such phenomena is paramount due to their wide range of applications. For ultrathin metallic films applied in micro-electronics, catalysis, or soft x-ray optics, small changes in the composition of surface and near-surface regions may lead to dramatic differences in properties ${ }^{1-5}$. Regarding applications of thin oxide films, such as protective layers against corrosion ${ }^{6}$, insulating layers in microelectronic ${ }^{7}$, and in catalytic devices ${ }^{8,9}$, control of synthesis conditions is often crucial to achieve optimal properties of oxide layers. Therefore, both for preventing oxidation of materials and for synthesis of oxide films, knowledge on fundamental oxygen-solid interaction processes is required. For the past years, this importance has been recognized and significant attempts have been made to study the initial oxidation of clean and well-prepared surfaces under ultrahigh vacuum (UHV) conditions both experimentally ${ }^{10,11}$ and theoretically ${ }^{12,13}$.

Nevertheless, accurate thickness and composition measurements on such thin films are not trivial. Techniques such as Rutherford backscattering spectrometry (RBS) or Secondary Ion Mass Spectroscopy (SIMS) are insufficiently precise for measuring the formed oxide thickness, due to limited resolution ${ }^{14,15}$. Angle-resolved X-ray spectroscopy (AR-XPS), X-ray reflectivity (XRR) or ellipsometry provide more precise thickness determination. However, these methods cannot be used for characterizing details of the kinetics of oxide growth, as they are not suitable for the tracing of marker isotopes. In this scenario, Low Energy Ion Scattering (LEIS) appears to be a unique tool for oxidation kinetics analysis. LEIS is a characterization technique known for its monolayer information depth ${ }^{15-17}$. Allied to this, its ability to differentiate atomic mass makes it a valuable tool for isotopic tracing experiments, especially for O-18 tracing in oxides ${ }^{15,18}$. A feature gaining more attention in the past years is the non-destructive in-depth analysis of sample composition, the so-called LEIS static depth profile (LEIS static DP). LEIS static DP is based on the analysis of signals from (mostly $\mathrm{He}^{+}$) projectiles scattered by sub-surface atoms that are reionised upon leaving the sample. This process produces a background signal, commonly called tail, that provides in-depth distribution of a material. This tail starts at energies just below the LEIS characteristic binary collision peak and extends down to a low-energy threshold. This threshold varies depending on the iontarget combination, allowing a maximum probed depth of 5-10 nm ${ }^{15}$. This technique is classified as static depth profiling as it provides in-depth information without damaging the sample, provided that low ion doses are 
applied ${ }^{15,17}$.

In this work we demonstrate that the change in background signal following oxygen exposure can also be applied for thickness determination of the oxide grown on the metal. Therefore, LEIS provides the opportunity to understand oxide growth mechanisms and kinetics by correlating the precise atomic identification of the outermost layer with the grown oxide thickness determined by static DP. Thereby, precise information regarding oxygen-solid interaction processes in nanoscaled systems can be acquired. We analyze the oxidation kinetics of ultrathin transition metal films of $\mathrm{Ru}, \mathrm{Ta}, \mathrm{Zr}$ and Mo by applying LEIS static DP and LEIS isotope tracing techniques. The films were deposited via DC-magnetron sputtering and transferred to analysis and oxidation chambers without any break of vacuum. The data obtained by static DP was correlated to traditional thickness-determination techniques, such as $\mathrm{XRR}$ and sputter depth profiling. Isotope tracing provided information on the diffusing species during oxide growth, corroborating the oxidation mechanism of the analyzed metals. We provide a detailed analysis of the oxidation mechanism and growth kinetics of metals exposed to atomic oxygen, highlighting the differences in behavior compared to oxidation by molecular oxygen, as obtained in previous experiments reported in the literature. Our results clearly demonstrate that the use of LEIS was critical in the understanding of important aspects previously neglected in experimental oxidation kinetics analysis.

\section{Experimental set-up and methods}

The experiments were performed in a home-designed ultrahigh vacuum system at a base pressure of $\leq 1 \times 10^{-9}$ mbar, which allows in-vacuum transfers between deposition (magnetron sputtering), characterization (LEIS) and oxygen exposure chambers with negligible surface contamination. Films of $\mathrm{Zr}$, Ta, Mo and $\mathrm{Ru}$ were deposited onto natively oxidized super-polished $\mathrm{Si}$ substrates by DC magnetron sputtering at room temperature, using $\mathrm{Ar}$ as working gas with a deposition pressure of $5 \times 10^{-4} \mathrm{mbar}$ and growth rate of typically $0.05 \mathrm{~nm} / \mathrm{s}$. The films were exposed at room temperature to neutral atomic oxygen species (O-16 or O-18) generated by a Specs MPS-ECR mini plasma source. The exposure time ranged from 1 to 240 minutes, at an atomic oxygen flow in the order of $10^{15}$ atoms $/ \mathrm{cm}^{2} / \mathrm{s}$ (partial pressure of $1 \times 10^{-4}$ 
mbar - background $\mathrm{O}_{2}$ ), exposing the entire sample.

The evolution of oxide thickness with exposure time was obtained by LEIS static depth profiling (static DP), with details described in subsection 2.1. LEIS measurements were performed in an ION-TOF GmbH Qtac ${ }^{100}$ high sensitivity LEIS spectrometer, described in details elsewhere ${ }^{19}$. $\mathrm{A} \mathrm{He}^{+}$ion beam at $3 \mathrm{keV}$ energy and 2.5-3.5 nA current, measured before each spectra acquisition in a Faraday cup, was chosen for characterization. During the measurement, the beam is rastered over a $1 \times 1 \mathrm{~mm}^{2}$ area, with typical ion dose density of $2 \times 10^{14} \mathrm{He}^{+}$ions $/ \mathrm{cm}^{2}$, and total spectrum acquisition time of 3 minutes. Using these analysis parameters, the dose density is low enough to stay below the so-called quasi-static limit ${ }^{20}$, for which artifacts due to ioninduced sputtering and intermixing are negligible, since the probability that a detected ion interacted with a sample site damaged by other ions from the same measurement is negligible. For the isotope tracing experiments, the metals were consecutively exposed to atomic O-16 and O-18 (isotopic enrichment 98\%) and analyzed by LEIS sputter depth profiling (sputter DP). In this analysis mode, each sputter step is followed by the acquisition of a LEIS spectrum under the same analysis conditions as previously described. Sputtering was done with a sputter gun attached to the LEIS chamber, positioned at $59^{\circ}$ with respect to the sample's surface normal, applying a 0.5 $\mathrm{keV} \mathrm{Ar}^{+}$beam at a $100 \mathrm{nA}$ average current. The sputtered area was fixed at $(2 \times 2) \mathrm{mm}^{2}$. The sputtered depth was calculated by verifying the necessary ion dose (at the specified energy and current) to sputter through a reference oxide sample with known thickness (determined by X-ray reflectometry).

To verify the accuracy of the oxide thickness determination via static DP, as well as the composition of the formed oxides, ex-situ X-ray reflectometry (XRR) was performed. The XRR measurements were obtained from a PANalytical Empyrean X-ray diffractometer ( $\mathrm{Cu}-\mathrm{Ka}$ radiation, $0.154 \mathrm{~nm})$.

\subsection{Methodology of oxide thickness determination by LEIS static depth profiling}

Static depth profiling is based on the analysis of the background (tail) signal in a LEIS spectrum. In LEIS, a sample is bombarded by noble gas ions with energies between 0.5 and $10 \mathrm{keV}$. In this energy regime, the projectiles are scattered from surface atoms almost exclusively by binary collisions, forming 
a characteristic Gaussian-shaped peak centered at an energy unique to each element ${ }^{16}$. If the referent element is present within the sample, a background (or tail) at the low energy side of its binary collision peak will emerge. The tail is formed by primary ions that penetrated into the solid, backscattered on a sub-surface atom and are emitted from the target in an ionized state into the direction of the analyzer. Due to the low energy applied in LEIS, noble gas ions are neutralized on penetrating the sample ${ }^{16,19,21,22}$. The formation of a tail will be determined by the finite probability of these scattered neutrals to be reionized upon leaving the sample, namely the reionization probability 16,23,24. This feature depends on the energy of the backscattered particle (i.e. its velocity) and surface atomic composition of the sample. Figure 1 shows examples of spectra obtained with $3 \mathrm{keV} \mathrm{He}^{+}$for both molybdenum and molybdenum oxide of different thicknesses. Only ions scattered from Mo can form the Mo related tail, as ions scattered from light elements lose more energy. Therefore, the ion yield energy in a LEIS tail signal depends both on the reionization probability and the distribution of the respective atoms inside the film ${ }^{16,19}$. Due to the stopping of particles by the target, these backscattered ions will present an energy loss with respect to the energy of the surface peak. On average, the longer the path travelled by the particle through the solid, the larger the energy loss will be. This feature allows the energy scale of the spectra to be translated into a depth scale [17]. For the geometry of our experiment, the depth $x$ from which a projectile is backscattered is found by the relation stated in Eq. (1) ${ }^{25,26}$ :

$$
x=\frac{E_{p}-E_{x}}{2.2 \cdot S}
$$

where $E_{p}$ is the energy of the relative binary collision peak, $E_{x}$ a specific energy in the tail signal, $S$ the stopping of ions by the target and the constant 2.2 is derived from the instrument geometry ${ }^{23,25,27}$. 

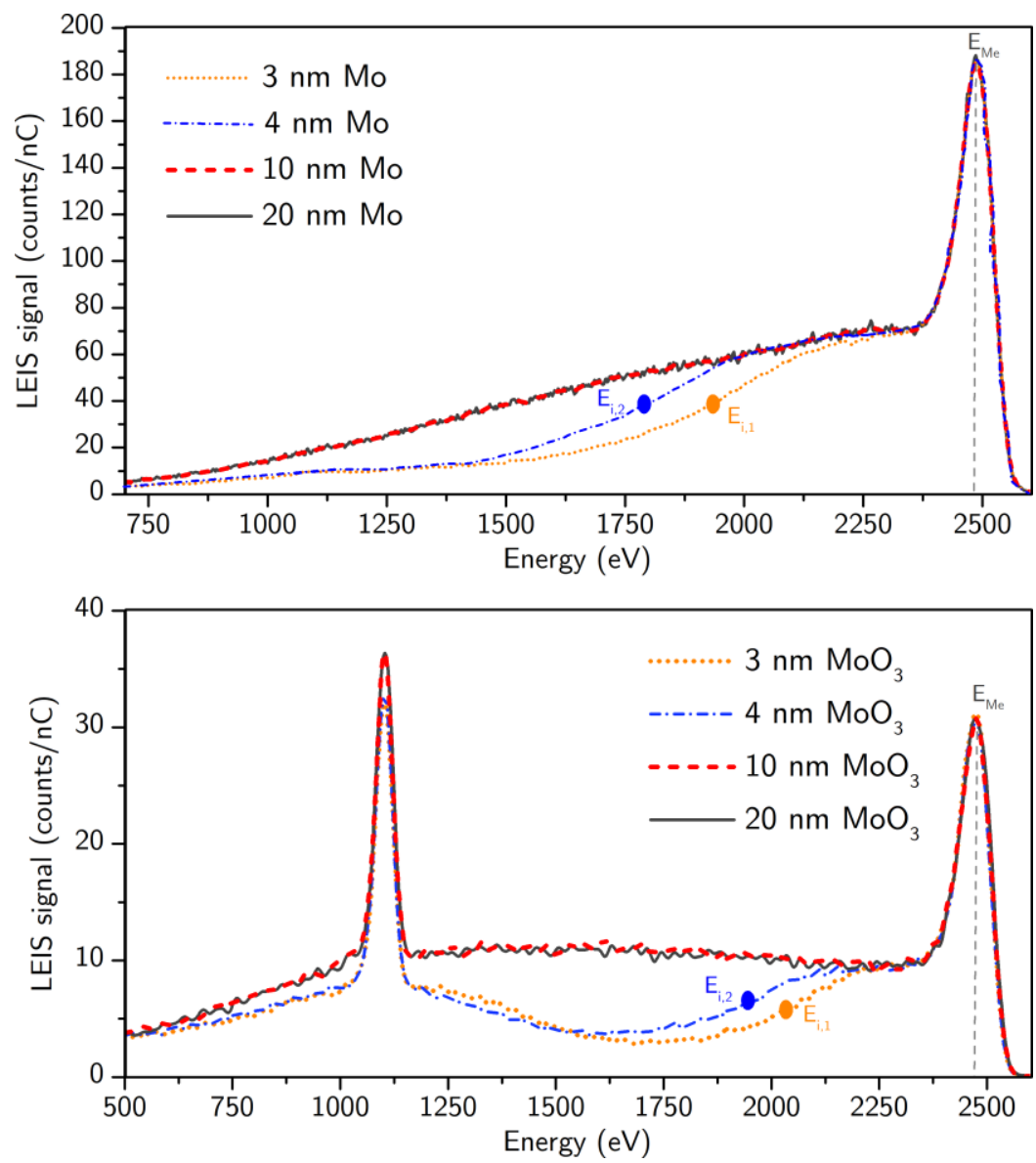

Figure 1: Demonstration of LEIS signals for 3, 4, 10 and $20 \mathrm{~nm}$ of molybdenum (top) and molybdenum oxide (bottom) deposited on a $\mathrm{Si}(100)$ substrate. $\mathrm{E}_{M e}$ represents the surface peak energy of the Mo metal and $\mathrm{E}_{i}$ the energy of the fitted error function inflection point (see Eq. 2).

Assuming the reionization probability to be constant for a given surface, the intensity of the tail can be applied for determining the in-depth distribution of atoms, as, for example, in determining the interface transition between two materials of different compositions 10,17,23,25. The interface between two layers is not expected to be sharp due to intermixing and interface roughness. In addition, straggling effects due to the stochastic nature of ion stopping will result in a smooth intensity transition between regions with different composition. Therefore, the interface can be modelled as an error function described in Eq. (2) ${ }^{25}$ :

$$
c=\frac{1}{2}\left(1-\operatorname{erf}\left(\frac{x-d}{\sqrt{2} \sigma}\right)\right)(2)
$$


where $c$ is the atom fraction at a given depth $x, d$ is the depth of the error function inflection point and $\boldsymbol{\sigma}$ is the width of the corresponding error function. However, one must be aware that the height and width obtained by a fit according to this equation are known to be affected by matrix and straggling effects, respectively. The straggling is a result of the statistical fluctuation of the energy-loss processes that particles are subjected to as they travel through matter ${ }^{28}$. The matrix effect appears from changes in neutralization according to changes in the composition of the material ${ }^{16,19,29}$. The position of the inflection point is the only variable minimally affected by artifacts ${ }^{25}$. Assuming the inflection point as an approximate limit between layers, its energy value can be applied as $E_{x}$ in Eq. (1) for determining the thickness of a sample. Examples of fitted inflection points are schematized in Figure 1 for $\mathrm{Mo}$ and $\mathrm{MoO}_{3}$. It is important to note that in the case of Mo and $\mathrm{MoO}_{3}$, for thicknesses higher than $10 \mathrm{~nm}$, the tail shape becomes constant. The limit for thickness detection emerges from the applied ion energy and stopping power of the target, this analysis can be typically applied for layer of thicknesses up to $10 \mathrm{~nm}^{21,26}$.

Analogously, the same procedure can be applied for determining the thickness of an oxide growing on a metal layer. Figure 2 shows an example of spectra evolution with oxide growth on a Mo film, where $E_{M e}$ is the surface peak energy of the Mo metal. It is observed that, following oxygen exposure, there is a decrease of the background intensity just below $E_{M e}$. This change can be interpreted as a decrease of metal concentration, a consequence of oxide formation. Therefore, an error function can be fitted in this region and the grown oxide thickness extracted. It should be noted that the metal concentration increases from oxide to metal, so the concentration will present an inverse profile compared to a metal film on a substrate with lighteratoms. Therefore, the complementary error function is applied in the fitting. With this, the energy value of the complementary error function inflection point, as schematized in Figure 2 by $E_{i}$, is applied in Eq. (1), and the grown oxide thickness on a metal is calculated. There may be a very slight difference in surface peak areas of Mo and $\mathrm{O}$ for the different $\mathrm{O}$ exposure times shown in Figure 2. This is possibly related to hydrogen contamination during or after atomic $\mathrm{O}$ exposure. However, this does not influence the determination of the error function inflection point and metal surface peak position. 


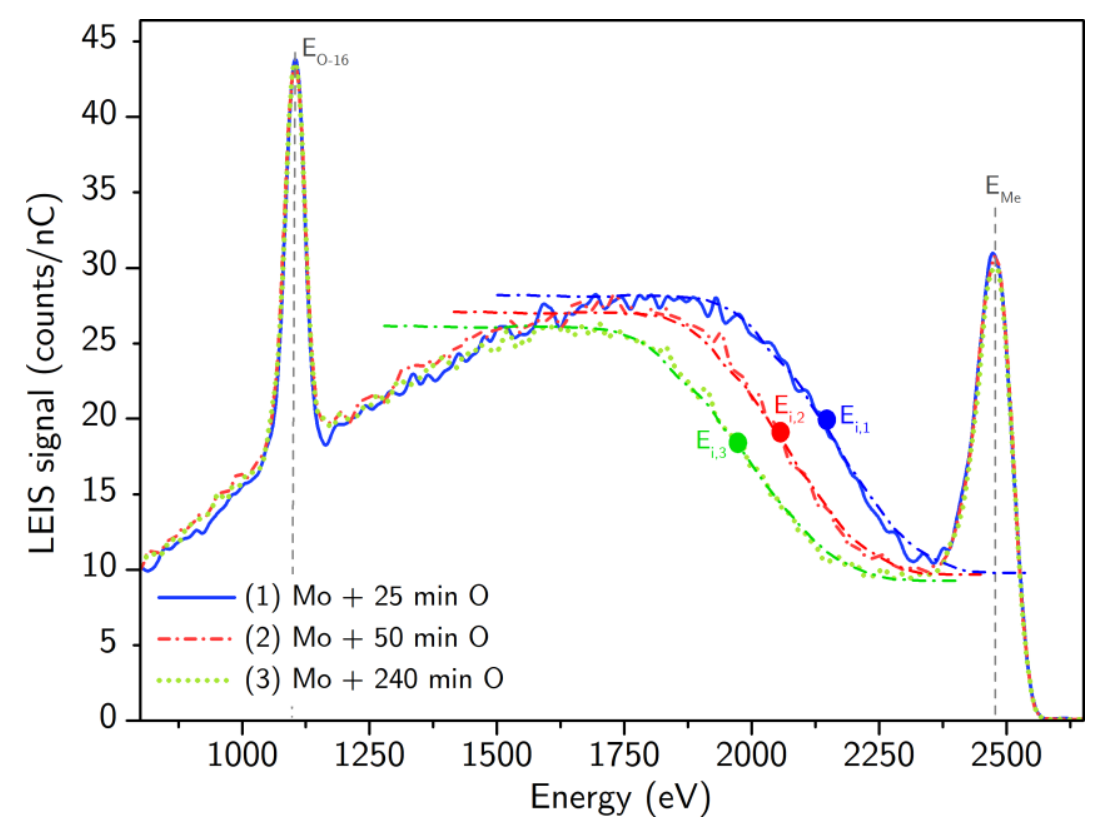

Figure 2: Demonstration of LEIS signal evolution with increasing of atomic $\mathrm{O}$ exposure time for Mo and parameters extracted from data for oxide thickness calculation.

Table 1: Values for metal surface peak energy $\left(E_{M e}\right)$ and stopping power $(S)$ applied for oxide thickness calculations. The stopping power values were obtained by SRIM software ${ }^{30}$.

\begin{tabular}{ccc}
\hline System & $\mathrm{S}[\mathrm{eV} / \mathrm{nm}]$ & $\mathrm{E}_{\mathrm{Me}}[\mathrm{eV}]$ \\
\hline $\mathrm{ZrO}_{2} / \mathrm{Zr}$ & 68.7 & 2470 \\
$\mathrm{Ta}_{2} \mathrm{O}_{5} / \mathrm{Ta}$ & 65.4 & 2680 \\
$\mathrm{MoO}_{3} / \mathrm{Mo}$ & 62.3 & 2490 \\
$\mathrm{RuO}_{2} / \mathrm{Ru}$ & 69.8 & 2515 \\
\hline
\end{tabular}

For the calculations performed in this paper, the stopping power was assumed to be equal to that of the bulk oxide form of each metal, as obtained from SRIM software ${ }^{30}$. The metal surface peak and inflection point energies were obtained by respectively fitting a Gaussian and a complementary error function in the respective spectrum regions with ION-TOF SurfaceLab 6 software. The values of the constants $E_{M e}$ and $S$ applied to calculations are listed in Table 1.

\section{Results and discussion}

Figure 3 shows the oxide thickness as a function of the exposure time 
obtained by static DP, XRR and LEIS sputter DP for $20 \mathrm{~nm}$ deposited metal films. Before exposure, the samples were analyzed with LEIS, confirming no prior oxygen adsorption. The error margin in the static DP arises from the uncertainty in stopping power that is inherent to the software calculation (3.5 percent of the obtained stopping for incident $\mathrm{He}^{+}$on targets) ${ }^{30}$, the fitting of the error function to the spectrum and standard errors. Errors in sputter DP are also derived from standard errors.

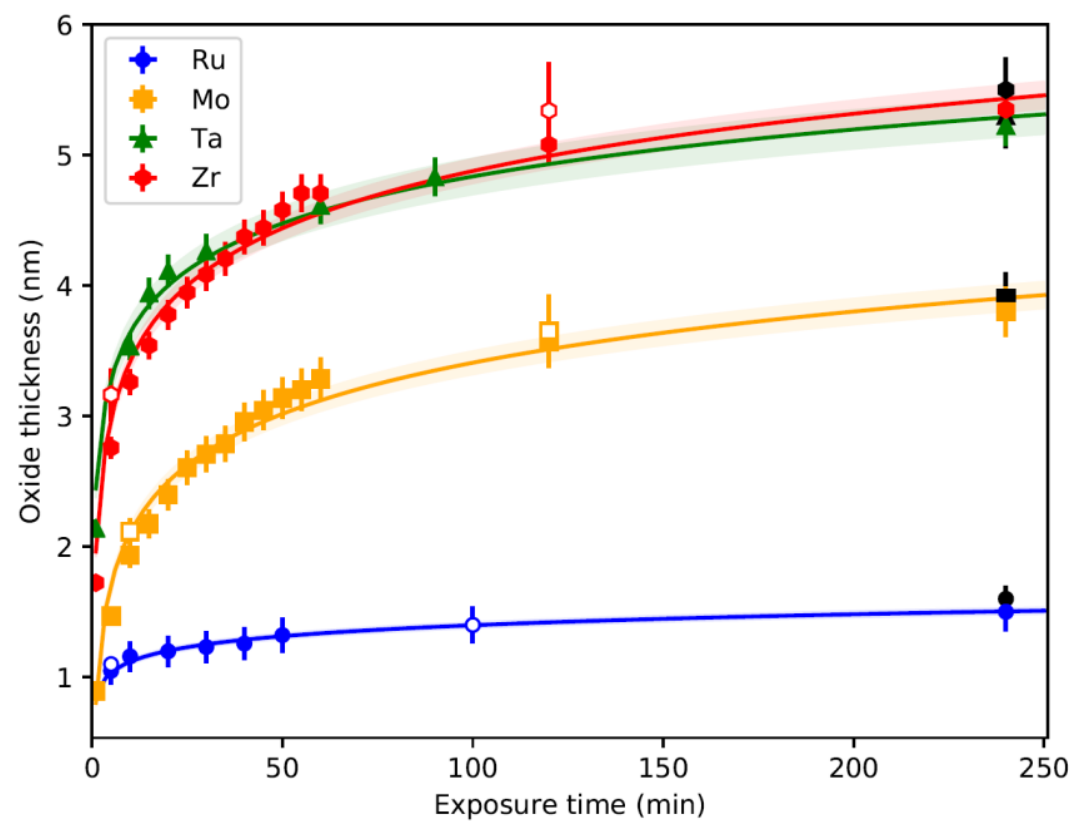

Figure 3: Oxide thicknesses derived from static DP versus atomic oxygen exposure time for Ru (blue circles), Mo (yellow squares), Ta (green triangles) and $\mathrm{Zr}$ (red hexagons), from 1 to 240 min exposure. Reference measurement by XRR (black symbols at $240 \mathrm{~min}$ ) and sputter profiles (open symbols) are given. The lines refer to the theoretically fitted growth curves based on the Cabrera-Mott inverse logarithm law. The shadowed area along the fits correspond to the fitting error.

After $240 \mathrm{~min}$ of exposure to atomic oxygen, the samples were removed from the vacuum system and directly analyzed by XRR. The layer thicknesses and associated errors were determined by fitting the obtained spectra with GenX software ${ }^{31}$. In GenX, a layered model of the structure is applied for simulating the reflection spectrum. For these samples, a pre-defined model was composed of the $\mathrm{Si}$ substrate and $\mathrm{SiO}_{2}$ layer of fixed thicknesses and densities, covered by the metal and a corresponding stoichiometric oxide layer. The free parameters applied in the GenX fitting were thickness and roughness of metal and oxide. Considering the oxide growth process $4,13,32,33$ 
and to minimize the differences between simulations and experimental data, an intermediate higher density oxide layer, which can be considered as a substoichiometric oxide, had to be added to the model, resulting in structures as shown schematically in Figure 4 with the corresponding thicknesses specified in Table 2. Figure 3 plots the sum of both stoichiometric and high density oxide layers for XRR values.

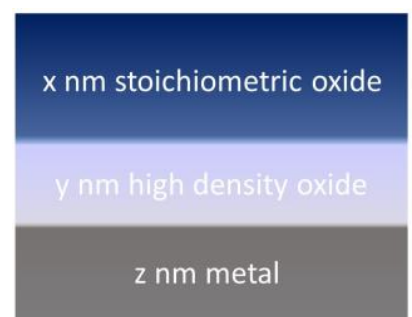

Figure 4: Model applied for XRR spectra simulation on GenX software.

Table 2: LEIS static DP and XRR obtained oxide thicknesses for saturated samples (240 min exposure to atomic $\mathrm{O}$ ). XRR values are obtained by a fit according to the model schematized in Figure 4.

\begin{tabular}{cccc}
\hline System & $\begin{array}{c}\text { Static DP } \\
{[\mathrm{nm}]}\end{array}$ & $\begin{array}{c}\text { Stoich. oxide } \\
{[\mathrm{nm}]}\end{array}$ & $\begin{array}{c}\text { XRR } \\
\text { High density oxide } \\
{[\mathrm{nm}]}\end{array}$ \\
\hline $\mathrm{Zr}$ & $5.1 \pm 0.2$ & $4.6 \pm 0.2$ & $0.9 \pm 0.2$ \\
$\mathrm{Ta}$ & $5.0 \pm 0.2$ & $3.6 \pm 0.3$ & $1.7 \pm 0.3$ \\
$\mathrm{Mo}$ & $3.6 \pm 0.2$ & $3.3 \pm 0.3$ & $0.6 \pm 0.3$ \\
$\mathrm{Ru}$ & $1.5 \pm 0.1$ & $1.3 \pm 0.1$ & $0.2 \pm 0.1$ \\
\hline
\end{tabular}

It is important to note that the tail analysis by LEIS does not allow for the identification of the two-layer oxide, as observed with XRR. This limitation comes from straggling effects suffered by scattered particles ${ }^{28}$. As mentioned in section 2.1, the stochastic nature of the stopping of ions by the target will lead to a smooth intensity transition between regions of different composition, which does not allow a deconvolution of possible in-depth regions of oxide with different stoichiometry. However, the agreement between the total oxide thickness obtained by XRR, the oxygen-containing thickness obtained by sputter depth profiling and the results obtained by static DP validate the use 
of LEIS for determining metal film oxidation in a fast and non-destructive manner. This is particularly important for the analysis of nanometric films, in which small changes in the composition of surface and near-surface regions may lead to dramatic differences in properties from optical ${ }^{1-3}$ to catalytic activity ${ }^{34}$. To further explore the application of the LEIS static DP technique, films of $3 \mathrm{~nm}$ Mo and $3 \mathrm{~nm} \mathrm{Zr}$ were exposed to atomic oxygen and analyzed by the presented method. A reactively deposited $1.5 \mathrm{~nm} \mathrm{ZrO}_{2}$ film, with deposition parameters described in Ribera et al. ${ }^{35}$, deposited on $20 \mathrm{~nm} \mathrm{Zr}$ also underwent the same experimental procedure. The resulting oxide growth profiles are shown in Figure 5.

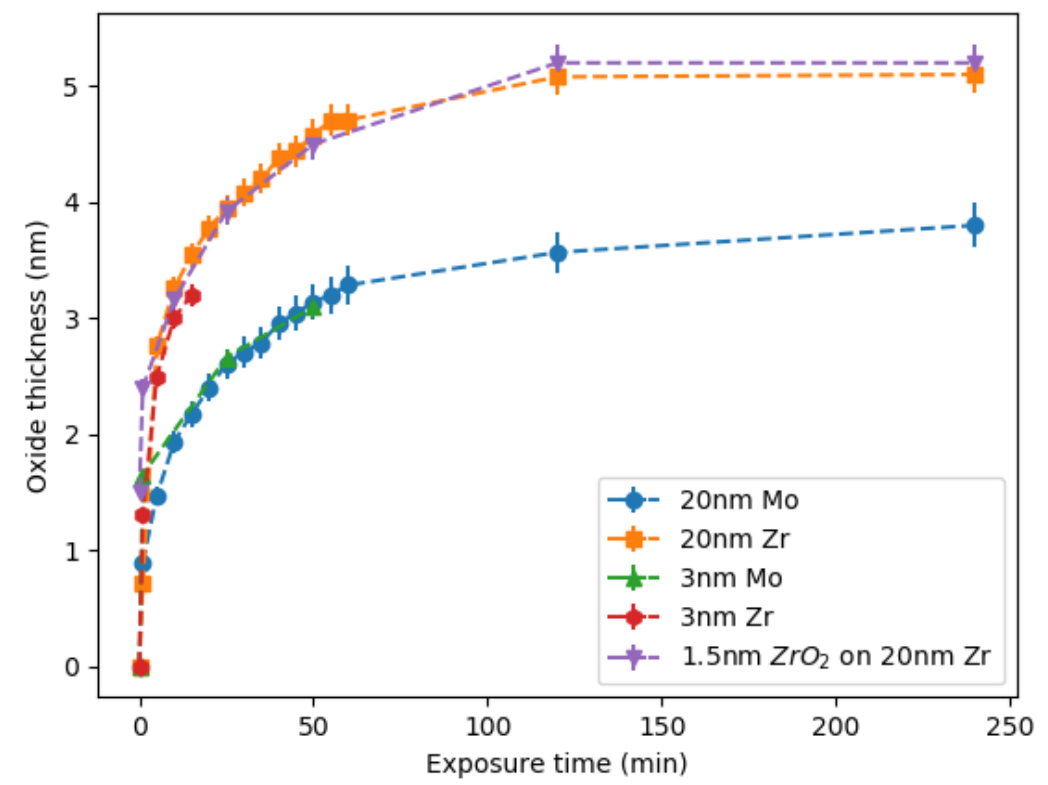

Figure 5: LEIS static DP oxide thicknesses for $20 \mathrm{~nm} \mathrm{Zr;} 1.5 \mathrm{~nm}$ sputter-deposited $\mathrm{ZrO}_{2}$ on 20 $\mathrm{nm} \mathrm{Zr;} 3 \mathrm{~nm} \mathrm{Zr;} 20 \mathrm{~nm}$ Mo and $3 \mathrm{~nm}$ Mo, all exposed to atomic oxygen from 1 min up to 240 min. The dashed lines serve as guides to the eye.

In both Figures 3 and 5, the oxidation profiles derived from static DP show that all metals present a rapid initial oxide growth, followed by a decrease in rate with increasing exposure time, until a stable oxide thickness is reached (limiting oxide thickness). This oxidation behavior is in line with the theory initially derived by Cabrera and Mott ${ }^{33}$ and further developed by Fromhold ${ }^{38}$. According to this theory, the activation energy for diffusion is overcome by a surface-charge field, which arises as a result of an electrostatic potential, also termed the Mott-potential, between the Fermi level of the metal and the electron acceptor levels of adsorbed oxygen species ${ }^{33,38,39}$. While the potential 
is constant, the formed field will be inversely proportional to the oxide thickness and, therefore, in the initial stages will have a high value ${ }^{37}$. This strong electric field promotes positive metal ion diffusion to react with the adsorbed oxygen, forming a new metal oxidelayer at the outer surface ${ }^{40}$. For our exposure conditions at room temperature, the Nernst-Einstein relation ( $q E_{M} a r k T$, with $E_{M}$ the field, $q$ the charge of ions, $a$ the ionic jump distance (which is in the same order as the lattice parameter), $k$ the Boltzmann constant and $T$ the temperature) is not met. This means that diffusion of metal or oxygen ions by the available thermal energy is insignificant compared to the electrical work by the Mott-potential. Therefore interpretation of oxide growth by Wagner's theory or the parabolic law is not applicable for our oxidation conditions ${ }^{36,37}$. In Fig. 3, superimposed on the experimental staticDP data points are the theoretically fitted growth curves based on the CabreraMott inverse logarithm law ${ }^{33,41}$, which has the form:

$$
\frac{1}{x}=A-\ln (t) \quad(3)
$$

where $x$ is the oxide thickness, $t$ is the exposure time and $A$ is a constant that contains material characteristics and temperature ${ }^{33}$. It is observed that the goodness of fit support the Cabrera-Mott mechanism of oxide growth for the analyzed regime.

To further elucidate the growth mechanism of the oxide films, isotope tracing LEIS sputter DP was applied. Figure 6 shows the relative concentrations of oxygen isotopes for $20 \mathrm{~nm} \mathrm{Zr}$, Ta and Mo consecutively exposed during $10 \mathrm{~min}$ to $\mathrm{O}-16$ and $10 \mathrm{~min}$ to $\mathrm{O}-18$. These values are relative to the total concentration of oxygen found at the surface (i.e. the metal peak and possible other elements were not taken into account for quantification). This approach was followed in order to avoid misinterpretation related to preferential sputtering and a possible matrix effect present in transition metal oxides ${ }^{25}$. Ru tracer analysis was not possible due to rapid saturated oxide thickness ( $\sim 1 \mathrm{~min}$, Fig. 3). The tracing of oxygen species shown in Figure 6 further confirmed the assumption of field-driven oxide formation. The profiles show that the exposure to O-18 leads to growth of additional oxide and possibly to an exchange of existing oxygen on the surface, a topic that will be further investigated in a following publication. However, it is important to note that oxygen on the outermost layers of formed oxides correspond to the 
isotope specie to which the sample was last exposed to. Therefore, the predominant migrating defect type can be assumed to be oxygen vacancies or metal interstitials ${ }^{33,38}$.
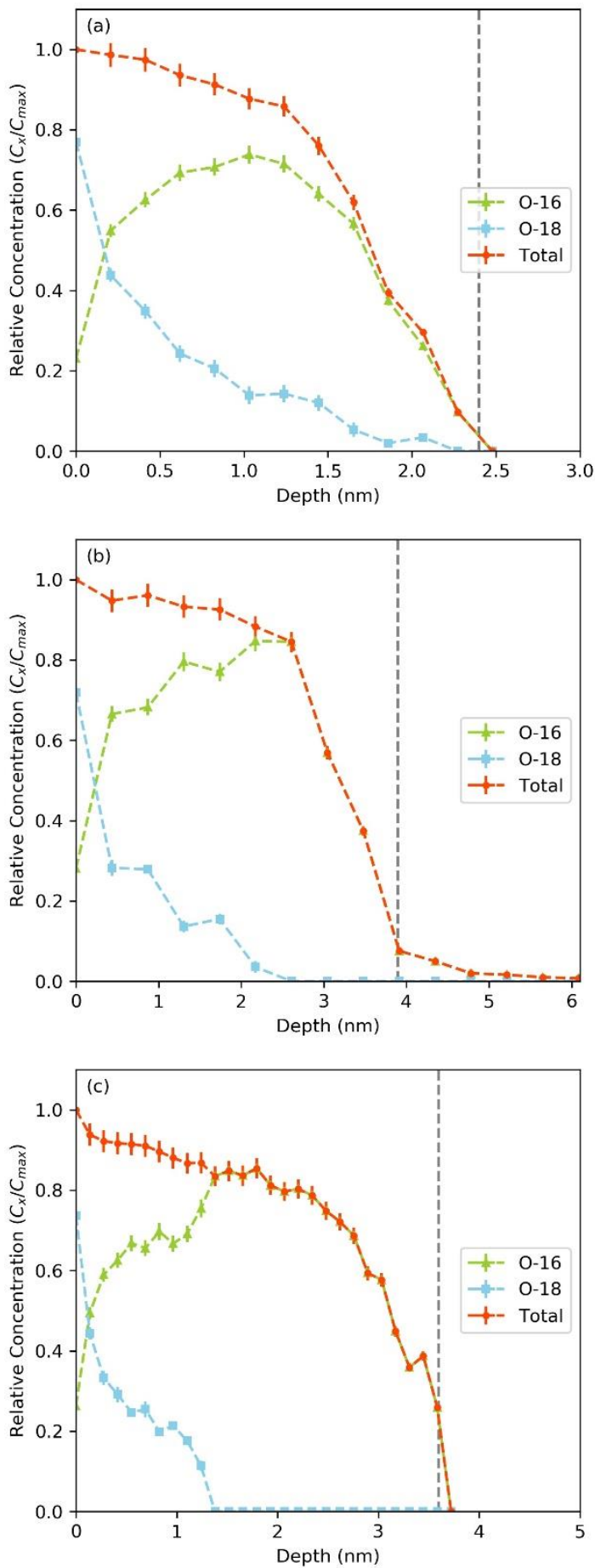

Figure 6: In-depth concentration of oxygen isotopes relative to the total O-16 + O-18 surface oxygen concentration for (a) Mo exposed 10 min to O-16 and 10 min to O-18, (b) Ta exposed 5 
min to $\mathrm{O}-16$ and $10 \mathrm{~min}$ to $\mathrm{O}-18$, and (c) $\mathrm{Zr}$ exposed during $10 \mathrm{~min}$ to $\mathrm{O}-16$ and $10 \mathrm{~min}$ to $\mathrm{O}-18$,. Samples were consecutively exposed to O-16 and O-18 with flow of $10^{15}$ atoms $/ \mathrm{cm}^{2} / \mathrm{s}$ (partial pressure of $110^{-4}$ mbar - background $\mathrm{O}_{2}$ ). The sputtered depth was calculated by verifying the necessary ion dose (at the specified energy and current) to sputter through a reference oxide sample with known thickness (determined by XRR). The gray dashed lines indicate the oxide thickness obtained by static DP.

One characteristic that should be noted is that the maximum oxide thicknesses achieved for exposures of thick metal films are considerably larger compared to those observed in natural oxidation; that is, the exposure of the metal to molecular oxygen at room temperature ${ }^{3,41,42}$. This can be explained by the absence of certain activated reaction steps for oxidation by atomic oxygen species. Oxidation by molecular oxygen at room temperature is described by three main steps: physisorption, dissociative chemisorption and potential set up ${ }^{42}$. Dissociative chemisorption leads to the accumulation of electron-acceptor species on the surface, which induce the Mott-potential to arise and the consequent incorporation of oxygen in the metal ${ }^{41,43}$. It has been shown both theoretically ${ }^{43}$ and experimentally ${ }^{41}$ that the concentration of oxygen species on a surface is determinant for the formation and maintenance of a Mott-field. In ambient $\mathrm{O}_{2}$, the potential set up may be kinetically hindered by the molecular dissociation step and the sticking probability of molecular oxygen on surfaces $40,42,44$. When atomic oxygen is used, these constraints do not exist. However, since these processes occur over a scale of just a few picoseconds, they are hardly observable through commonly available experimental techniques ${ }^{45}$. Simulations performed by Gibson et al. on $\mathrm{Rh}(111)$ indicated that atomic oxygen leads to the facile formation of a fullcoverage and ordered $(1 \times 1) \mathrm{O}$ monolayer, which induced $\mathrm{O}$ absorption into the bulk to proceed much more readily in comparison to oxidation by $\mathrm{O}_{2}$ 46 . The exclusion of the dissociation step from the reaction pathway also leads to laterally uniform oxide growth, as defects and irregularities at atomic and nano scales, which act as active sites for molecular dissociation, do not interfere in the accumulation of oxygen at the surface ${ }^{34,43,44,47-49}$. The results in Table 2 for XRR analysis of oxide-saturated films confirm the formation of an almost completely stoichiometric layer, including a small substoichiometric oxide interface. Therefore, direct use of atomic oxygen eliminates the dissociation step of $\mathrm{O}_{2}$ as reaction barrier and maintains a high concentration of strong electron acceptors in the surface. In this way, oxide growth will only cease when the field generated by the Mott-potential is insufficient to act as 
driving force for ionic diffusion through the oxide. We now investigate whether the Mott-Cabrera theory ${ }^{33}$ can be used to understand the differences in oxidation of the four analyzed metals. According to the theory, the Mottpotential is defined by Eq. (4):

$$
\mathrm{V}_{\mathrm{M}}=\mathrm{e}^{-1}\left(\varphi_{0}-\varphi_{\mathrm{L}}\right)(4)
$$

where $\boldsymbol{\Phi}_{0}$ is the metal work function, $\boldsymbol{\varphi}_{L}$ the difference between the vacuum potential and the Fermi level in the presence of adsorbed oxygen and $e$ the elementary charge. The lower the metal work function, the more negative the formed potential will be, and the higher the generated field $\left(E_{M}=-V_{M} / L(t)\right.$, where $\mathrm{L}(\mathrm{t})$ is the grown oxide thickness as a function of time $t$ ). This field then lowers the energy barriers for initiation of ionic motion. With the oxide reaching ahigh $L(t)$ value, the field will no longer be effective in decreasing the diffusion barrier, causing the oxide thickening to stop. Work function values for polycrystalline structures obtained from literature show the lowest values for $\mathrm{Zr}(4.0 \mathrm{eV})$ and $\mathrm{Ta}(4.0 \mathrm{eV})$, followed by $\mathrm{Mo}(4.3 \mathrm{eV})$ and $\mathrm{Ru}(4.7$ $\mathrm{eV})$. It is known that the value of the work function is dependent on the conditions of the surface (cleanliness, preferential plane), which is why measurements reported in the literature often cover a considerable range ${ }^{50}$. However, from the considered values, it is possible to correlate higher work function to a lower final oxide thickness as observed in this study.

In the case of oxidation by molecular oxygen, it is usually assumed that the cause of slow oxide film thickening is the formation of a closed oxide layer and consequent inhibition of electron transport from metal to adsorbed oxygen 5. However, in our experiments it is seen that even when a closed layer of oxide is pre-deposited on the metal surface by reactive sputtering $(1.5 \mathrm{~nm} \mathrm{ZrO} 2$ on $20 \mathrm{~nm} \mathrm{Zr}$ in Figure 5), the exposure to atomic oxygen induces the continuation of oxide growth with a similar growth rate, until a similar saturation thickness is reached. This result indicates that when in Cabrera-Mott growth regime, it is not the formation of a closed oxide layer, but the lower concentration of atomic oxygen species on the surface that diminishes the field and leads to lower self-limited oxide growth. This decrease in concentration may come from oxide surfaces having both lower reactivity for $\mathrm{O}_{2}$ bond dissociation and lower sticking probability of the molecule. This observation meets previous studies where the influence of photon incidence ${ }^{42,45}$, temperature and oxygen 
pressure ${ }^{41}$ were verified for field-induced oxidation. Cai et al. ${ }^{41}$ observed that the increase in $\mathrm{O}_{2}$ surface concentration, by lowering of temperature or increase of partial pressure, lead to thickening of self-limited oxide on $\mathrm{Al}(111)$. For $\mathrm{UV}$-assisted oxidation, the interaction of $\mathrm{UV}$ photons with $\mathrm{O}_{2}$ or $\mathrm{O}_{3}$ increases the molecule dissociation rate on oxide surfaces, and oxidation profiles analogous to the ones obtained in this paper are reached ${ }^{42,45}$. These observations indicate that in cases where the presence of atomic oxygen species on the surface is not hindered by dissociation or adsorption, the obtained limiting oxide thickness will be directly related to the metal work function and, consequently, the formed Mott-field.

\section{Summary}

The use of atomic oxygen for room temperature oxidation of various transition metal films was studied by low energy ion scattering analysis. A methodology for determining the grown oxide thickness via LEIS static depth profiling has been presented in detail. This non-destructive method agreed well with results obtained by XRR and sputter-depth profiling. Exposures of $20 \mathrm{~nm}$ deposited metal films yielded a limiting oxide thickness of around $5 \mathrm{~nm}$ for $\mathrm{Zr}$ and $\mathrm{Ta}$, while Mo and $\mathrm{Ru}$ formed an oxide thicknesses of $\sim 3.5 \mathrm{~nm}$ and $1.5 \mathrm{~nm}$, respectively. With the use of isotope tracing coupled with LEIS sputter-depth profiling it was possible to verify that the reaction front is located on the oxygen/oxide surface in all analyzed samples, with the dominant diffusing species being the metal interstitials or oxygen vacancies. These findings indicate that the oxide formation observed on metals by atomic $\mathrm{O}$ exposure at room temperature is primarily driven by the value of the metal work function $(\mathrm{Zr} \sim \mathrm{Ta}<\mathrm{Mo}<\mathrm{Ru})$, which will determine the Mott-potential intensity. The use of LEIS in oxidation analysis was valuable in the understanding of oxidation kinetics and mechanisms in the room temperature regime.

\section{Acknowledgements}

This work is part of HTSM project 13913, funded by NWO Applied and Engineering Sciences with co-funding by Carl Zeiss SMT. The authors also acknowledge the Industrial Focus Group XUV Optics at the MESA+ Institute 
at the University of Twente, as well as the Province of Overijssel.

\section{References}

${ }^{1}$ T.E. Madey, N.S. Faradzhev, B. V. Yakshinskiy, and N. V. Edwards, Appl. Surf. Sci. 253, 1691 (2006).

${ }^{2}$ F. Liu, J.M. Sturm, C.J. Lee, and F. Bijkerk, Surf. Sci. 646, 101 (2016).

${ }^{3}$ R. Müller, S. Yulin, P. Naujok, N. Kaiser, and A. Tünnermann, Thin Solid Films 624, 1 (2017).

${ }^{4}$ A.J. Samin and C.D. Taylor, J. Appl. Phys. 123, (2018).

${ }^{5}$ L. Nguyen, T. Hashimoto, D.N. Zakharov, E.A. Stach, A.P. Rooney, B. Berkels, G.E. Thompson, S.J. Haigh, T.L. Burnett, P. Aidan, and B. Berkels, ACS Appl. Mater. Interfaces 10, 2230 (2018).

6 G. Balakrishnan, T.N. Sairam, P. Kuppusami, R. Thiumurugesan, E. Mohandas, V. Ganesan, and D. Sastikumar, Appl. Surf. Sci. 257, 8506 (2011). ${ }^{7}$ S. Kumar, Z. Wang, X. Huang, N. Kumari, N. Davila, J.P. Strachan, D. Vine, A.L.D. Kilcoyne, Y. Nishi, and R.S. Williams, ACS Nano 10, 11205 (2016). ${ }^{8}$ I.A. de Castro, R.S. Datta, J.Z. Ou, A. Castellanos-Gomez, S. Sriram, T. Daeneke, and K. Kalantar-zadeh, Adv. Mater. 29, 1 (2017).

${ }^{9}$ D.R. Miller, S.A. Akbar, and P.A. Morris, Sensors Actuators, B Chem. 204, 250 (2014).

${ }^{10}$ Y. Wu, E. Garfunkel, and T.E. Madey, Surf. Sci. 365, 337 (1996).

${ }^{11}$ R. Steinberger, C.E. Celedón, B. Bruckner, D. Roth, J. Duchoslav, M. Arndt, P. Kürnsteiner, T. Steck, J. Faderl, C.K. Riener, G. Angeli, P. Bauer, and D. Stifter, Appl. Surf. Sci. 411, 189 (2017).

${ }^{12}$ M. Aykol and K.A. Persson, ACS Appl. Mater. Interfaces 10, 3039 (2018).

${ }^{13}$ V.P. Parkhutik, J. Phys. D. Appl. Phys. 25, 256 (1992).

${ }^{14}$ H.H. Strehblow, Electrochim. Acta 212, 630 (2016).

${ }^{15}$ J.A. Kilner, S.J. Skinner, and H.H. Brongersma, J. Solid State Electrochem. 15, $861(2011)$. 
${ }^{16}$ H.H. Brongersma, M. Draxler, M. de Ridder, and P. Bauer, Surf. Sci. Rep. 62, 63 (2007).

${ }^{17}$ M. De Ridder, R.G. Van Welzenis, H.H. Brongersma, and U. Kreissig, Solid State Ionics 158, 67 (2003).

${ }^{18}$ H.H. Brongersma, T. Grehl, P.A. van Hal, N.C.W.W. Kuijpers, S.G.J.J. Mathijssen, E.R. Schofield, R.A.P.P. Smith, and H.R.J.J. ter Veen, Vacuum 84, 1005 (2010).

19 A.A. Zameshin, A.E. Yakshin, J.M. Sturm, H.H. Brongerma, and F. Bijkerk, Appl. Surf. Sci. 440, 570 (2018).

${ }^{20}$ C. V. Cushman, P. Brüner, J. Zakel, G.I.H. Major, B.M. Lunt, T. Grehl, N.J. Smith, M.R. Linford, T. Grehl, and M.R. Linford, Anal. Methods 8, 3419 (2016)

${ }^{21}$ R. ter Veen, M. Fartmann, R. Kersting, and B. Hagenhoff, J. Vac. Sci. Technol. A Vacuum, Surfaces, Film. 31, 01 A113 (2013).

${ }^{22}$ R. Souda, K. Yamamoto, W. Hayami, T. Aizawa, and Y. Ishizawa, Surf. Sci. 363, 139 (1996).

${ }^{23}$ V.I.T.A. De Rooij-Lohmann, A.W. Kleyn, F. Bijkerk, H.H. Brongersma, and A.E. Yakshin, Appl. Phys. Lett. 94, (2009).

${ }^{24}$ M. Tsukada, S. Tsuneyuki, and N. Shima, Surf. Sci. 164, 811 (1985).

${ }^{25}$ A.A. Zameshin, Probing Atomic Scale Interface Processes Using X-Rays and Ions, University of Twente, 2018.

${ }^{26}$ S. Tsuneyuki and M. Tsukada, Phys. Rev. B 34, 5758 (1965).

${ }^{27}$ R.C. Ribera, R.W.E. Van De Kruijs, S. Kokke, E. Zoethout, A.E. Yakshin, and F. Bijkerk, Appl. Phys. Lett. 105, 13 (2014).

${ }^{28}$ W.K. Chu, Phys. Rev. A 13, 2057 (1976).

29 S.N. Mikhailov, L.C.A. van den Oetelaar, and H.H. Brongersma, Nucl. Instruments Methods Phys. Res. B 93, 210 (1994).

${ }^{30}$ J.F. Ziegler, M.D. Ziegler, and J.P. Biersack, SRIM - The Stopping and 
Range of Ions in Matter (Ion Implantation Press, 2008).

${ }^{31}$ M. Björck and G. Andersson, J. Appl. Crystallogr. 40, 1174 (2007).

${ }^{32}$ R.C. Ribera, R.W.E. van de Kruijs, A.E. Yakshin, and F. Bijkerk, J. Appl. Phys. 118, 055303(1 (2015).

${ }^{33}$ N. Cabrera and N.F. Mott, Rep. Prog. Phys 12, 163 (1949).

${ }^{34}$ B. Herd, M. Knapp, and H. Over, J. Phys. Chem. C 116, 24649 (2012).

${ }^{35}$ R. Coloma Ribera, R.W.E. Van de Kruijs, J.M. Sturm, A.E. Yakshin, and F. Bijkerk, J. Appl. Phys. 121, (2017).

${ }^{36}$ A. Atkinson, Rev. Mod. Phys. 57, 437 (1985).

${ }^{37}$ Z. Xu, K.M. Rossob, and S. Bruemmer, Phys. Chem. Chem. Phys. 14, 14534 (2012).

38 A.T. Fromhold, Theory of Metal Oxidation (North Holland Publishing Company, Amsterdam, 1976).

${ }^{39}$ L.P.H. Jeurgens, A. Lyapin, and E.J. Mittemeijer, Surf. Interface Anal. 38, 1380 (2006).

${ }^{40}$ O. Guise, J. Levy, and J.T. Yates, Thin Solid Films 496, 426 (2006).

${ }^{41}$ N. Cai, G. Zhou, K. Müller, and D.E. Starr, Appl. Phys. Lett. 101, 1 (2012).

${ }^{42}$ S. Ramanathan, D. Chi, P.C. McIntyre, C.J. Wetteland, and J.R. Tesmer, J. Electrochem. Soc. 150, F110 (2003).

${ }^{43}$ M. Todorova, W.X. Li, M. V. Ganduglia-Pirovano, C. Stampfl, K. Reuter, and M. Scheffler, Phys. Rev. Lett. 89, 096103 (2002).

${ }^{44}$ S.K.R.S. Sankaranarayanan and S. Ramanathan, J. Phys. Chem. C 2008, 112, 17877 (2008).

${ }^{45}$ M. Tsuchiya, S.K.R.S. Sankaranarayanan, and S. Ramanathan, Prog. Mater. Sci. 54, 981 (2009).

${ }^{46}$ K.D. Gibson, M. Viste, E.C. Sanchez, and S.J. Sibener, J. Chem. Phys. 110, 2757 (2002).

${ }^{47}$ P. Dudin, A. Barinov, L. Gregoratti, M. Kiskinova, F. Esch, C. Dri, C. 
Africh, and G. Comelli, J. Phys. Chem. B 109, 13649 (2005).

${ }^{48}$ A. Soon, M. Todorova, B. Delley, and C. Stampfl, Phys. Rev. B - Condens. Matter Mater. Phys. 73, 1 (2006).

${ }^{49}$ C. Stampfl, Catal. Today 105, 17 (2005).

${ }^{50}$ W.M. Haynes, CRC Handbook of Chemistry and Physics, 95th ed. (CRC Press, 2014). 